\title{
ANNOUNCEMENT
}

\section{VNS Young Investigator Award}

Dr. Stefanie Volland received the 2015 VNS Young Investigator Award at the biennial Biology and Chemistry of Vision Federation of American Societies for Experimental Biology (FASEB) Meeting in Big Sky, Montana. Her work was selected from among many excellent studies that were presented at this meeting. Dr. Volland is working on unraveling the detailed mechanisms of rod photoreceptor outer segment disk formation. To help address this question she employed serial electron tomography and 3D modeling to analyze the disk nanostructure at a $2 \mathrm{~nm}$ resolution in the $x, y$, and $z$-axis in several different mammals. Although technically difficult, the technique of serial electron tomography is key to understanding complex 3D structures. Dr. Volland's findings support a model of disk morphogenesis where the newly forming rod outer segment disks are initially open to the extracellular space, although the process is somewhat more complex than depicted in previously proposed models. Dr. Volland conducted these studies in the laboratory of Dr. David S. Williams at the Jules Stein Eye Institute at the University of California, Los Angeles.

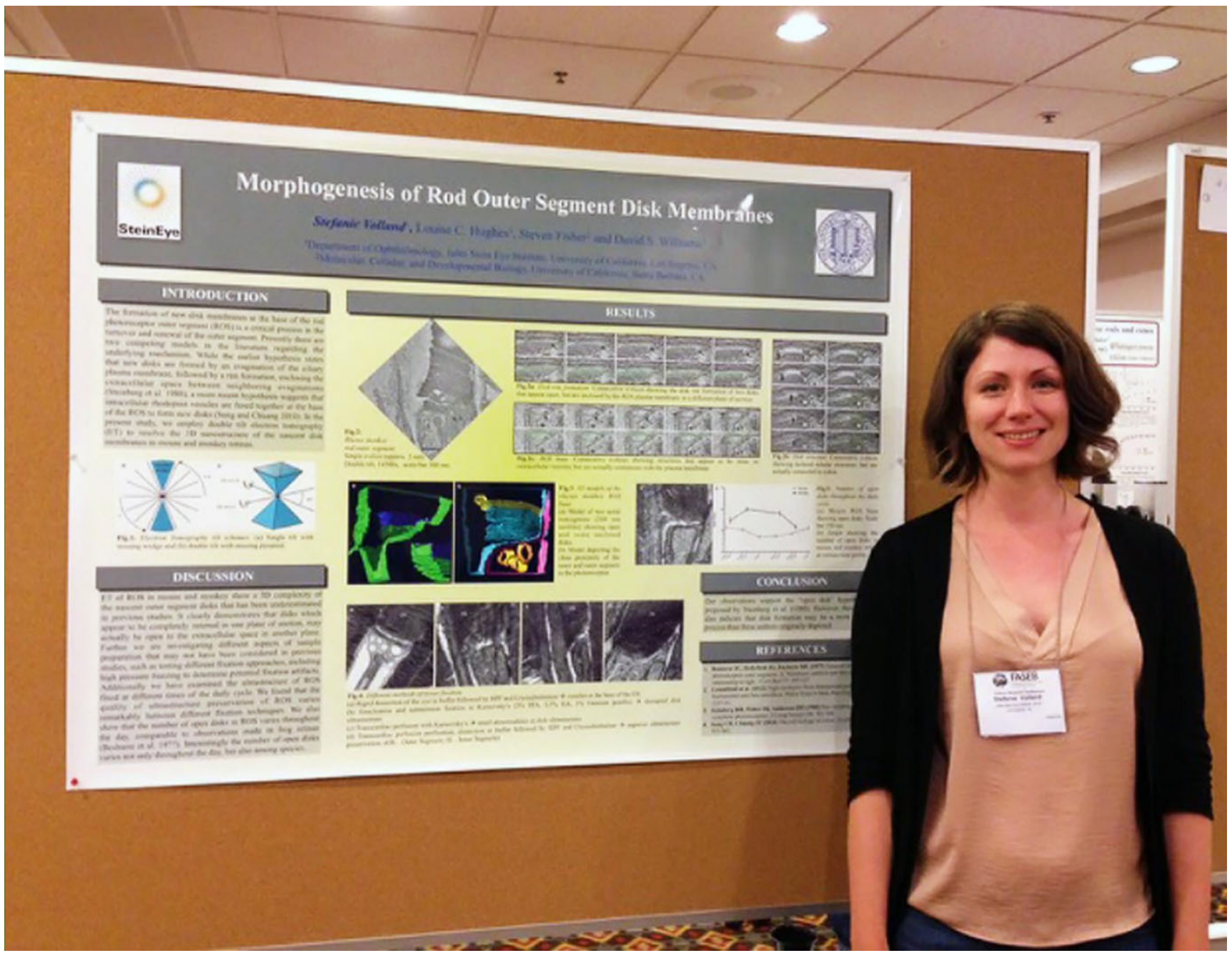

